\title{
Internalizing and Externalizing Problem Behaviours and Their Relations with Self-Concept and Parental Psychological Aggression among Junior Middle School Students
}

\author{
Wang Yaqun \\ School of Education, Tianjin University \\ Tianjin, 300350, P.R.China
}

\begin{abstract}
The present study aimed to investigate the mediating effect of adolescent self-concept in the relationship between parental psychological aggression and problem behaviours. The youth self-report, wallace self-concept scale and psychological aggression scale were conducted on 286 junior middle school students. Results were as follows: (1)The level of internal problem behavior of girls in grade 9 is higher than that of girls in grade 7 and of boys in grade 9. (2) Students' selfconcept was negatively correlated with internalizing and externalizing problem behaviours and parental psychological aggression, while internalizing and externalizing problem behaviours were positively correlated with parental psychological aggression, respectively. (3)Self-concept played a partial mediation role in the relationship between maternal psychological aggression and internalizing and externalizing problem behaviours, whereas it played full mediation role in the relationship between paternal psychological aggression and externalizing problem behaviours. These findings indicated that the self-concept mediated the relationship between parental psychological aggression and problem behaviours.
\end{abstract}

Keywords-Junior middle school students; Psychological aggression; Self-concept; Internalizing and externalizing problem behaviours

\section{INTRODUCTION}

The problem behaviour is mainly manifested as internalizing problem behaviour and externalizing problem behavior. Internalizing problem behaviour refers to the internal psychological problems, including anxious/depressed, withdrawn/depressed, and somatic complaints [1]. Externalizing behaviour refers to external disorders, including rule-breaking behaviour and aggressive behaviour. Junior middle school students are in the puberty. And puberty is also a vulnerable period of internalizing and externalizing problem behaviours. Internalizing and externalizing problem behaviours during adolescence would persist into adulthood, resulting in academic failure, depression, suicide and crime [2]. Therefore, it is an important research topic in the field of clinical psychology to explore the characteristics of internalizing and externalizing behaviour of junior middle school students and the prevention and intervention measures.
Psychological aggression (PA) is operationally defined as "the use of psychological force with the intention of causing a child to experience psychological pain or fear for the purposes of correction or control of misbehavior," e.g., "threatened to spank or hit" [3]. Numerous studies have demonstrated that parental Psychological aggression (PA) may lead to Junior middle school students' internalizing and externalizing problem behaviors [4]. The studies evaluating potential longterm effects of parental psychological aggression in adolescence has identified several negative effects including anxiety, physical aggression, bullying behavior [5]. In addition, parental psychological aggression can also affect young people's self-concept [6]. However, the self-concept is closely related to the internalizing and externalizing problem behaviours. For example, self-concept was negatively correlated with anxiety, depression, aggression and violence. Studies have shown that self-concept mediates the relationship between parenting and problem behavior [7]. Accordingly, we speculate that parental psychological aggression may affect junior middle school students' problem behavior by influencing their self-concept.

Based on the above analysis, three hypotheses were proposed:

H1. There will be significant gender and grade differences in junior middle school students' internalizing and externalizing problem behaviours.

$\mathrm{H} 2$. There will be significant correlations between problem behavior and self-concept, parental psychological aggression.

H3. self-concept will mediate the relationship between parental psychological aggression and internalizing and externalizing problems, separately. 


\section{METHOD}

\section{A. Participants}

Participants in the current study were 286 junior middle school students $(52.8 \%$ male), recruited from Shandong Province in China. Participants ranged from 12-15 years of age and were enrolled in grades 7-9. The proportion of participants living in towns and cities was $3.85 \%$ and $96.15 \%$, respectively.

\section{B. Measures}

Psychological Aggression Scale (PA). The 22-item Chinese version of Parent-Child Conflict Tactics Scales (CTSPC) is used to assess parents' disciplinary practices. The CTSPC can be broken down into the following subscales: Nonviolent Discipline (four items), Psychological Aggression (five items), Corporal Punishment (six items), Severe Physical Abuse (three items), and Very Severe Physical Abuse (four items). Variables for PA in the current study are established and defined by specific CTSPC items of the PA subscales. The respondent reports separate frequencies for CTSPC items to indicate how often their mother and father engaged in each strategy at about the time the respondent was age 13 or whether the strategy was used prior to age 13. Participants responded using the following scale: once (1); twice (2); 3 to 5 times (4); 6 to 10 times (8); 11 to 20 times (15); more than 20 times (25); never (0); not at age 13, but it happened before (1). In the current study, Cronbach's alphas for the PA were 0.81 .

Wallace Self-Concept Scale (WSCS). The scale consists of 15 Likert-scale items. From "warm" to "indifferent" are recorded 1-7 points, respectively. Six items need to reverse the score. The higher the total score, the higher the level of self- concept. Internal consistency for this measure was adequate in the current sample ( $\alpha=0.94)$.

Youth Self-Report (YSR). Using the Youth Self-Report Scale (2001), which comes from Achenbach's Child Behavior Checklist (CBCL). The scale is suitable for measuring the internalizing and externalizing problem behaviours of adolescents aged 11 to 18 years old. The scale is made up of 53 Likert items scored on a 0-2 Likert scale. The internal consistency of this study was adequate on a general level and across the different subscales (internalizing problem behaviour's $\alpha=0.94$, externalizing problem behaviour's $\alpha$ $=0.93$ ).

\section{PROCEDURE}

All participants were volunteers. Participants completed the PA, WSCS, and YSR in a 20-minute period. The distribution order of the questionnaires was randomly varied. The procedure was explained to each participant and informed consent forms were signed.

\section{RESULTS}

\section{A. Descriptive statistics}

Descriptive statistics for PA variables used in the analyses are shown in Table 1.

TABLE I MEANS AND STANDARD DEVIATIONS OF INTERNALIZING AND EXTERNALIZING PROBLEM BEHAVIOURS

\begin{tabular}{|c|c|c|c|c|c|c|c|}
\hline \multicolumn{2}{|c|}{} & \multicolumn{2}{c|}{ Grade7 } & \multicolumn{2}{c|}{ Grade8 } & \multicolumn{2}{c|}{ Grade9 } \\
\cline { 3 - 8 } & & Boys & girls & Boys & girls & Boys & girls \\
\hline \multirow{3}{*}{ internalizing } & $\bar{x}$ & 7.47 & 6.21 & 8.92 & 8.47 & 7.02 & 11.82 \\
\cline { 2 - 8 } & $s$ & 7.38 & 4.95 & 7.39 & 7.22 & 8.88 & 11.24 \\
\hline \multirow{2}{*}{ externalizing } & $\bar{x}$ & 5.96 & 4.40 & 8.88 & 5.21 & 6.40 & 8.35 \\
\cline { 2 - 8 } & $s$ & 7.28 & 4.35 & 9.87 & 4.95 & 10.65 & 11.09 \\
\hline
\end{tabular}

The variance analysis of $3 \times 2$ was carried out by using internalizing and externalizing problem behaviours as the dependent variables and grade and gender as independent variables. The results showed that the interaction effect of internalizing problem behaviour was significant in gender and grade $(\mathrm{F}(2,286)=3.92, \eta 2=0.03, \mathrm{P}<0.05)$. Further simple effect analysis found that girls in grade 9 reported higher level of internalizing problem behaviour than girls in grade 7 and boys in grade 9 .

B. Relations between PA, self-concept, and internalizing and externalizing problem behaviours

Bivariate correlations among all primary variables are presented in Table 2 . The correlation matrix indicated that selfconcept was negatively correlated with internalizing and externalizing problem behaviours and parental psychological aggression, while internalizing and externalizing problem behaviours were positively correlated with parental psychological aggression, respectively. 
TABLE II CORRELATIONS BETWEEN PA, SELF-CONCEPT, AND INTERNALIZING AND EXTERNALIZING PROBLEM BEHAVIOURS

\begin{tabular}{|c|c|c|c|c|}
\hline & 1 & 2 & 3 & 4 \\
\hline 1 internalizing problem & & & & \\
\hline 2 externalizing problem & $0.63^{* *}$ & & & \\
\hline 3 self-concept & $-0.65^{* *}$ & $-0.54^{* *}$ & & \\
\hline 4 paternal aggression & $0.56^{* *}$ & $0.55^{* *}$ & $-0.57^{* *}$ & $0.76^{* *}$ \\
\hline 5 maternal aggression & $0.59^{* *}$ & $0.58^{* *}$ & $-0.61^{* *}$ & $0.05 \%$, \\
\hline
\end{tabular}

\section{Mediation model}

The structural equation model was used to investigate the relationship between parental psychological aggression, selfconcept and internalizing and externalizing problem behaviours (see Fig. 1). The model showed a good fit: $\chi^{2} / \mathrm{df}=3.63$, RMSEA=0.096, SRMR=0.066, CFI=0.941, TLI=0.912. Selfconcept mediated the relationship between parental psychological aggression and internalizing and externalizing problems separately.

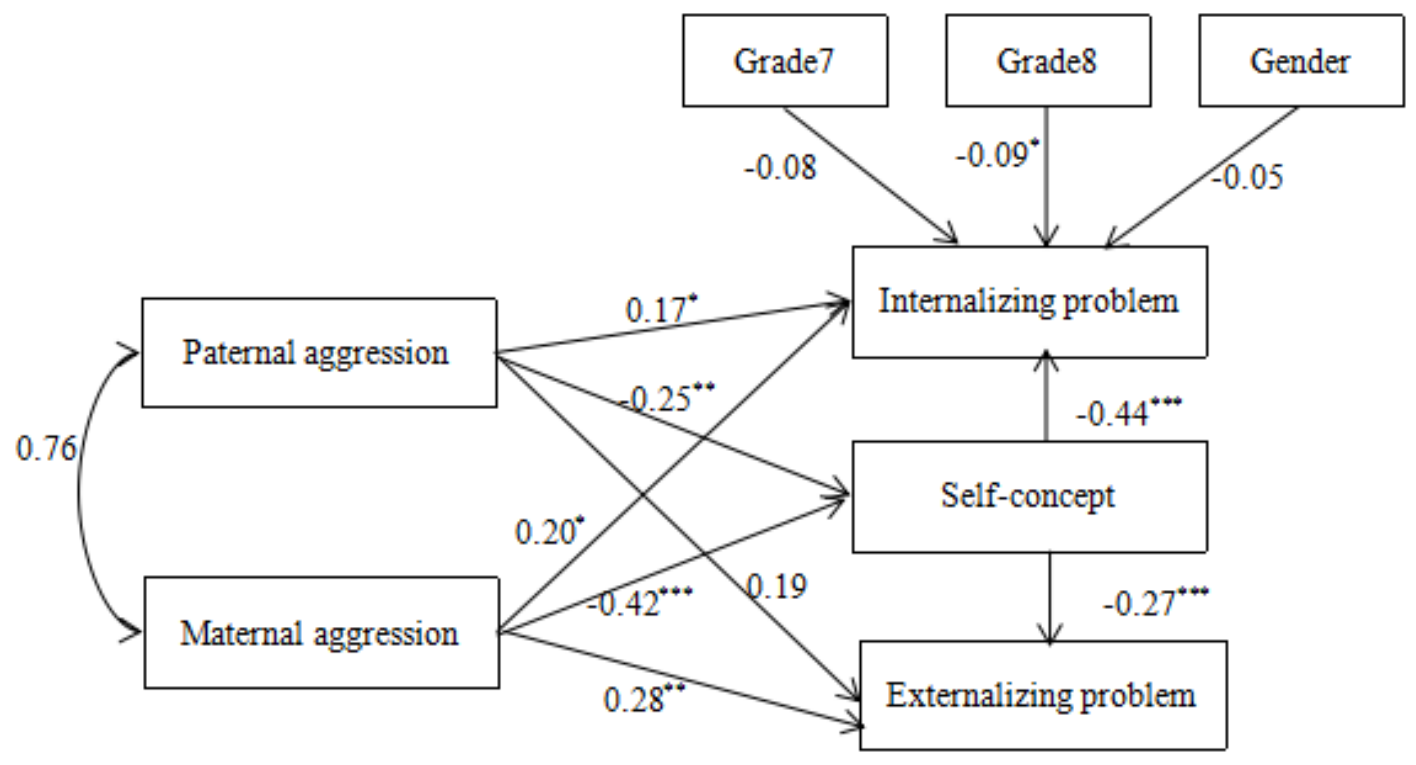

Fig. 1 Mediation model of self-concept

\section{DISCUSSION}

This study found that girls in grade 9 reported higher level of internalizing problem behaviour than girls in grade 7 and boys in grade 9 . It is possible that puberty development lead to the emergence of this result. In puberty, the secretion of sex hormones increases with age, so girls on the stress and emotional susceptibility increase [8]. There is a significant gender difference in the developmental status of puberty. Girls due to the sudden rise in female hormones often in a higher state of emotional awakening. Therefore, compared with boys, girls are more sensitive in the emotional aspect [9]. Simultaneously, the process of socialization of girls is more passive than boys. Parents and teachers tend to strengthen the girls with their internalizing behavior to express their feelings, especially negative feelings. Therefore, girls in the face of difficulties are more inclined to use internalizing problem behaviour to express their poorly adapted [9].
Maternal psychological aggression not only directly influenced problem behaviours, but indirectly influenced problem behaviours through self-concept; paternal psychological aggression directly influenced internalizing problem behaviour, but indirectly influenced internalizing and externalizing problem behaviours through self-concept. This may be due to the mother in the process of raising children to play a dominant position, is the main participant in the process of education. However, the time of contact between the father and the child is shorter and the scope of the contact is limited to learning and basic life.

The present study revealed the mediating effect of adolescent self-concept in the relationships between parental psychological aggression and internalizing and externalizing problem behaviours. Based on this result, this study suggests that parents should adopt more positive parenting such as encouragement, support, care and so on, reduce the negative parenting, and promote the formation of a positive selfcognition and self-acceptance of children. 


\section{ACKNOWLEDGMENT}

The authors would like to acknowledge Danhua Wang from No.12 Middle school of Linyi for her assistance in analyzing portions of the data reported in the current manuscript.

\section{REFERENCES}

[1] Xu, F., Zhang, L., Wei, X., et al. (2015). The stability of internalizing problem and its relation to maternal parenting during early adolescence. Psychological Development and Education, 31(2), 204-211. (In Chinese)

[2] Straus MA, Hamby SL, Finkelhor D, et al. Identification of child maltreatment with the Parent-Child Conflict Tactics Scales: Development and psychometric data for a national sample of American parents. Child abuse \& neglect, 1998, 22(4): 249-270.

[3] Barajas-Gonzalez RG, Brooks-Gunn J. Income, neighborhood stressors, and harsh parenting: Test of moderation by ethnicity, age, and gender. Journal of family psychology, 2014, 28(6): 855.

[4] Wang M, Wang X, Liu L. Paternal and maternal psychological and physical aggression and children's anxiety in China. Child abuse \& neglect, 2016, 51: 12-20.

[5] Gã ${ }^{3}$ Mez-Ortiz O, Romera E M, Ortega-Ruiz R. Parenting styles and bullying. The mediating role of parental psychological aggression and physical punishment [J]. Child Abuse Negl, 2016, 51:132-143.

[6] McKinney C, Milone MC, Renk K. Parenting and late adolescent emotional adjustment: Mediating effects of discipline and gender. Child Psychiatry \& Human Development, 2011, 42(4): 463-481.

[7] Chen, C. The relationship among parenting styles, self-concept and social anxiety of the adolescents [D]. Master's thesis, Shanxi Normal University, 2015.

[8] Zhong, N., Ling, H. The effect of pubertal timing on adolescent depression and anxiety. Chinese Journal of Clinical Psychology, 2013, 21(5): 842-846

[9] Zhang, Z. The Relationship among Friendship Quality, Self-Concept and Depression: Comparison Between Healthy Adolescents and HearingImpaired Adolescents [D]. Master's thesis, Shandong Normal University, 2015. 